Trauma Berufskrankh 2012 · 14[Suppl 2]:194-196 DOI 10.1007/s10039-011-1765-5

Online publiziert: 3. September 2011

(c) Springer-Verlag 2011
N. Wischnewski

Gesundheitsamt Charlottenburg-Wilmersdorf, Berlin

\section{Hygiene in der Praxis} Aus der Praxis

\section{Hintergrund}

Die gesetzliche Grundlage für Praxisbegehungen durch das Gesundheitsamt ist das Infektionsschutzgesetz (IfSG; $\$ 36$ Absatz 1), in dem festgelegt ist, dass Einrichtungen zum ambulanten Operieren der infektionshygienischen Überwachung durch das Gesundheitsamt unterliegen. Weiterhin wird dort gefordert, dass diese Einrichtungen in Hygieneplänen die innerbetrieblichen Verfahrensweisen zur Infektionshygiene festlegen müssen. Zudem ist dort die fortlaufende Aufzeichnungspflicht von nosokomialen Infektionen und dem Auftreten von Krankheitserregern mit speziellen Resistenzen und Multiresistenzen festgelegt. Aufgabe des Gesundheitsamts ist es, Hygienepläne und Aufzeichnungen sowie deren Umsetzung vor Ort zu kontrollieren und die Praxisinhaber bei hygienerelevanten Fragestellungen zu beraten.

Als fachliche Basis für die Begehung werden die Empfehlungen der Kommission für Krankenhaushygiene und Infektionsprävention, angesiedelt beim Robert Koch-Institut (RKI), herangezogen, die für jeden kostenlos auf den Internetseiten des RKI abrufbar sind (- Infobox 1). Insbesondere die Empfehlungen

- Anforderungen der Hygiene bei Operationen und anderen invasiven Eingriffen,

- Anforderungen der Hygiene beim ambulanten Operieren in Krankenhaus und Praxis,

- Prävention postoperativer Infektionen im Operationsgebiet,

- Händehygiene,
- Anforderungen an die Hygiene bei der Reinigung und Desinfektion von Flächen und

- Anforderungen an die Hygiene bei der Aufbereitung von Medizinprodukten

sind für die ambulant operierende Praxis von Bedeutung.

Bei der Erstellung eines Hygieneplans ist v. a. die individuelle Anpassung der Empfehlungen an örtliche Gegebenheiten wichtig. Im Rahmen der Begehungen wird darauf geachtet, ob die im Hygieneplan beschriebenen Abläufe mit dem Praxisalltag kompatibel sind und umgesetzt werden.

Merke. In der Hygiene gibt es keinen Unterschied zwischen ambulanten und stationären Eingriffen.

\section{Anforderungen der Hygiene bei Operationen und anderen invasiven Eingriffen}

In dieser Empfehlung wird der Gefährdungsgrad für operative Eingriffe hinsichtlich einer möglichen Infektion unterschieden. Dabei dienen die Art des Eingriffs (Operation/kleinere invasive Eingriffe/invasive Untersuchungen) und der Kontaminationsgrad des Operationsgebiets als Grundlage. Die hygienischen Anforderungen richten sich somit nach der Aufgabenstellung des Operateurs und sollen gleichzeitig eine adäquate Raumplanung für sinnvolle Abläufe und hygienisch einwandfreies Arbeiten berücksichtigen.

\section{Baulich-funktionelle Anforderungen für den Operationsbereich}

Nach den Empfehlungen der Kommission für Krankenhaushygiene ist der Operationsbereich (OP-Bereich) vom übrigen Praxisbereich $\mathrm{zu}$ trennen. $\mathrm{Zu}$ einem OPBereich gehören:

Personalumkleideräume mit einer klaren Trennung zwischen reiner und unreiner Seite. Die Bevorratung des Tagesbedarfs an reiner OP-Kleidung erfolgt auf der reinen Seite, die unreine Seite dient zur Ablage der Stationskleidung, beinhaltet einen Sammelbehälter für benutzte OP-Kleidung und ein WC.

Operationsraum/-räume. Diese sind in sich abgeschlossen, haben wenig Türen sowie keine Wasserarmaturen und Bodenabläufe, und ihre Oberflächen, Einbauten und Geräte sind leicht zu reinigen und zu desinfizieren. Da die Raumluft als Infektionsquelle eine untergeordnete Bedeutung bei postoperativen Wundinfektionen hat, wird sie für aseptische Eingriffe mit besonders hohem Infektionsrisiko für erforderlich angesehen.

\section{Infobox 1 Internetlinks}

- Empfehlungen der Kommission für Krankenhaushygiene und Infektionsprävention des RKI: http://www.rki.de $\rightarrow$ Infektionsschutz $\rightarrow$ Krankenhaushygiene

- Referenzdaten des Nationalen Referenzzentrums für Surveillance: http://www. nrz-hygiene.de 
Waschplatz und die Möglichkeit der Händedesinfektion. Sie müssen außerhalb des OP-Raums liegen. Der Wasserhahn soll ohne Handkontakt (Einhebelmischbatterie) bedienbar sein und je einen Spender für Händedesinfektionsmittel und Waschlotion sowie einen Handtuchspender aufweisen (TRBA 250).

Weitere Anforderungen. Im OP-Bereich sind ein Lager für Sterilgut, saubere Geräte und Medikamente (Kühlschrank), ein Bereich für die Narkoseein- und -ausleitung, ein unreiner Arbeitsraum zur Aufbereitung, ein Entsorgungsraum für unreine Güter, ein Raum für Putzmittel sowie ggf. ein Patientenumkleide- und ggf. ein Aufwachraum vorzuhalten.

Eingriffsraum. Er benötigt im Unterschied zum OP-Bereich nur einen Umkleidebereich für das Personal mit der Möglichkeit zur Händedesinfektion und zur Entsorgung. Es sollen eine Fläche für die Aufbereitung von Geräten und ggf. für Lagerung, Entsorgung und Aufbereitung von Geräten bzw. Verbrauchsmaterial sowie ggf. eine Umkleidemöglichkeit und ein Ruheraum für Patienten vorhanden sein.

\section{Betrieblich-organisatorische Anforderungen}

Die innerbetrieblichen Verfahrensweisen werden praxisspezifisch im Hygieneplan festgelegt ( $\$ 36$ IfSG). Dabei müssen die Qualitätsanforderungen und Prozessabläufe/Aufgabenspektren berücksichtigt werden. Der Gesetzgeber macht keine konkreten Vorgaben für den Aufbau und den Inhalt eines Hygieneplans, da die Individualität einer Praxis im Vordergrund stehen soll. Vorgefertigte Pläne (z. B. von Desinfektionsmittelherstellern) können als Orientierungshilfe dienen, sind aber allein als nicht ausreichend anzusehen, da sie meist allgemein gehalten sind, um möglichst viele Praxisbereiche abdecken zu können.

Surveillance. Sie zählt zu den betrieblich-organisatorischen Anforderungen und wird nach dem Infektionsschutzgesetz $\$ 23$ auch für die ambulant Operierenden gefordert. Sie dient der internen

Trauma Berufskrankh 2012 · 14[Suppl 2]:194-196 DOI 10.1007/s10039-011-1765-5

(C) Springer-Verlag 2011

\section{N. Wischnewski}

\section{Hygiene in der Praxis. Aus der Praxis}

\section{Zusammenfassung}

Einrichtungen zum ambulanten Operieren unterliegen laut Infektionsschutzgesetz (IfSG) der infektionshygienischen Überwachung durch das Gesundheitsamt. Dieses kommt der Aufgabe durch Praxisbegehungen, Kontrolle der Hygienepläne und Aufzeichnungen sowie Beratung des Praxisinhabers zu hygienerelevanten Fragestellungen nach. Wichtig ist, stets zu berücksichtigen, dass es in der Hygiene keinen Unterschied zwischen ambulanten und stationären Eingriffen gibt. Die Empfehlungen der Kommission für Krankenhaushygiene und Infektionsprävention beim Robert Koch-Institut (RKI) lassen sich kostenlos auf den Internetseiten des
RKI abrufen. Im vorliegenden Beitrag werden für Praxen, in denen ambulant operiert wird, wichtige baulich-funktionelle sowie betrieblich-organisatorische Aspekte vorgestellt. Abschließend wird beispielhaft auf das praktische Vorgehen des Gesundheitsamts Charlottenburg-Wilmersdorf bei der infektionshygienischen Überwachung eingegangen.

\section{Schlüsselwörter}

Ambulante Operation .

Infektionsschutzgesetz · Hygiene ·

Infektionshygienische Überwachung .

Gesundheitsamt

\section{Hygiene in the medical practice. From experience}

\begin{abstract}
According to the German Protection Against Infection Act, facilities for outpatient surgery are subject to supervise by the Health Authority to monitor hygienic conditions regarding infectious agents. This task is fulfilled by inspecting the practice premises, checking the hygiene plans and records, and advising the practice owner on questions relevant to hygiene. It is important that consideration always be given to ensuring that there are no differences in hygiene adherence between inpatient and outpatient interventions. The recommendations of the Commission on Hospital Hygiene and Infection Prevention of the Robert Koch Institute can
\end{abstract}

be downloaded free of charge from their website. This contribution presents constructional/ functional and operational/organizational aspects important for practices offering outpatient surgeries. The article closes with an example of how the Health Authority of Charlottenburg-Wilmersdorf conducts the surveillance of hygiene and infection control.

\section{Keywords}

Ambulatory surgical procedures . Protection Against Infection Act · Hygiene · Infection control · Health Authority
Qualitätssicherung und bietet, bei Verwendung einheitlicher Kriterien (CDCKriterien, CDC: „Centers for Disease Control“), eine anonyme Vergleichbarkeit zu den Referenzdaten des Nationalen Referenzzentrums für Surveillance ( $\boldsymbol{\bullet}$ Infobox 1).

\section{Praktisches Vorgehen des Gesundheitsamts Charlottenburg-Wilmersdorf}

Bevor eine Begehung durch das Gesundheitsamt erfolgt, wird der Praxisinhaber mit einem Anschreiben und Hinweis auf die Rechtsgrundlage schriftlich informiert und um eine Terminabsprache gebeten. Dem Anschreiben wird eine Checkliste, die vorab ausgefüllt werden soll und baulich-funktionelle sowie betrieblich-organisatorische Aspekte beinhaltet, beigefügt. Zudem erfolgt die Bitte zur Übersendung des Hygieneplans, um diesen im Vorfeld begutachten zu können.

Bei der Begehung vor Ort erläutert der Praxisbetreiber die Schwerpunkte seiner operativen Tätigkeit, und der Amtsarzt kann den vorab durchgesehenen Hygieneplan hinsichtlich der Situation vor Ort prüfen. Hierbei wird das Augenmerk auf hygienerelevante Abläufe in der Praxis, wie Umgang mit Mehrdosisbehältnissen, die Vorbereitung von Spritzen, die Möglichkeiten und Indikationen zur Händehygiene und die Aufbereitung von Medizinprodukten, gelegt. 


\section{Hygiene in der Praxis - aus der Praxis}

Im Anschluss daran wird der Weg des Patienten vom Betreten bis zum Verlassen der Praxis unter hygienerelevanten Punkten abgegangen. Hierbei werden die Betriebs-, Funktions- und Aufbereitungsräume unter den Aspekten der baulichfunktionellen Anordnung und der technisch-apparativen Ausstattung betrachtet und die jeweiligen betrieblich-organisatorischen Abläufe beurteilt. Im Anschluss daran werden in einer Nachbesprechung die Ergebnisse und Probleme erörtert und ein Bericht mit ggf. Auflagenbescheid und Terminsetzung zur Mängelbeseitigung schriftlich übersandt. Gegebenenfalls erfolgt eine erneute Überprüfung der Praxis, um die Umsetzungen der Mängelbeseitigung nachzuvollziehen.

\section{Fazit für die Praxis}

Das Infektionsschutzgesetz, § 36 Absatz 1 , ist die rechtliche Grundlage für Begehungen durch das Gesundheitsamt. Hierbei dienen die Empfehlungen der Kommission für Krankenhaushygiene und Infektionsprävention, angesiedelt beim Robert Koch-Institut, als wissenschaftliche Basis für die Bewertung.

Hygienepläne sollen der individuellen Situation einer Praxis gerecht werden und bei Begehungen durch das Gesundheitsamt in ihrer Struktur erkennbar und umsetzbar sein.

Die durch den Gesetzgeber geforderte Surveillance kann einen internen Verlaufsüberblick geben und ist zusammen mit der Erregerstatistik zu bewerten. Das moderne Gesundheitsamt ist für hygienische Fragestellungen gern ein unabhängiger Berater, der sich nicht nur über die Aufgaben der Kontrollfunktion definiert, sondern kompetente Hilfestellung und individuelle Lösungsmöglichkeiten bieten kann.

\section{Korrespondenzadresse}

\section{N. Wischnewski}

Gesundheitsamt Charlottenburg-Wilmersdorf, Hohenzollerndamm 174-177, 10713 Berlin nwischnewski@yahoo.de

Interessenkonflikt. Der korrespondierende Autor gibt an, dass kein Interessenkonflikt besteht. 